\title{
Divertor Target Heat Load Reduction by Electrical Biasing, and Application to COMPASS-D
}

S.J. Fielding, R.H. Cohen, P. Helander and D.D. Ryutov

This article was submitted to European Physical Society, Budapest, Hungary, June 12-16, 2000

\section{March 7, 2001}

Lawrence

Livermore

National

Laboratory 


\section{DISCLAIMER}

This document was prepared as an account of work sponsored by an agency of the United States Government. Neither the United States Government nor the University of California nor any of their employees, makes any warranty, express or implied, or assumes any legal liability or responsibility for the accuracy, completeness, or usefulness of any information, apparatus, product, or process disclosed, or represents that its use would not infringe privately owned rights. Reference herein to any specific commercial product, process, or service by trade name, trademark, manufacturer, or otherwise, does not necessarily constitute or imply its endorsement, recommendation, or favoring by the United States Government or the University of California. The views and opinions of authors expressed herein do not necessarily state or reflect those of the United States Government or the University of California, and shall not be used for advertising or product endorsement purposes.

This is a preprint of a paper intended for publication in a journal or proceedings. Since changes may be made before publication, this preprint is made available with the understanding that it will not be cited or reproduced without the permission of the author.

This report has been reproduced directly from the best available copy.

Available electronically at http://www.doc.gov/bridge

Available for a processing fee to U.S. Department of Energy

And its contractors in paper from

U.S. Department of Energy

Office of Scientific and Technical Information

P.O. Box 62

Oak Ridge, TN 37831-0062

Telephone: (865) 576-8401

Facsimile: (865) 576-5728

E-mail: reports@adonis.osti.gov

Available for the sale to the public from

U.S. Department of Commerce

National Technical Information Service

5285 Port Royal Road

Springfield, VA 22161

Telephone: (800) 553-6847

Facsimile: (703) 605-6900

E-mail: orders@ntis.fedworld.gov

Online ordering: http:/ / www.ntis.gov/ordering.htm

\section{OR}

Lawrence Livermore National Laboratory

Technical Information Department's Digital Library

http: / / www.llnl.gov/tid/Library.html 


\title{
Divertor target heat load reduction by electrical biasing, and application to COMPASS-D
}

\author{
S.J.Fielding, R.H. Cohen ${ }^{a}$, P.Helander and D.D. Ryutov ${ }^{a}$ \\ EURATOM/UKAEA Fusion Association, Culham Science Centre, Abingdon, Oxfordshire, OX14 3DB, UK \\ ${ }^{a}$ Lawrence Livermore National Laboratory, Livermore, USA
}

\begin{abstract}
A toroidally-asymmetric potential structure in the scrape-off layer (SOL) plasma may be formed by toroidally distributed electrical biasing of the divertor target tiles. The resulting ExB convective motions should increase the plasma radial transport in the SOL and thereby reduce the heat load at the divertor [1]. In this paper we develop theoretical modelling and describe the implementation of this concept to the COMPASS-D divertor. We show that strong magnetic shear near the X-point should cause significant squeezing of the convective cells preventing convection from penetrating above the $\mathrm{X}$-point. This should result in reduced heat load at the divertor target without increasing the radial transport in the portion of the SOL in direct contact with the core plasma, potentially avoiding any confinement degradation. Implementation of divertor biasing is in hand on COMPASS-D involving insulation of, and modifications to, the present divertor tiles. Calculations based on measured edge parameters suggest that modest currents $\sim 8 \mathrm{~A}$ tile are required, at up to $150 \mathrm{~V}$, to drive the convection. A technical test is preceeding full bias experiments.
\end{abstract}

\section{Introduction}

The avoidance of high heat loading on divertor target surfaces is one of the key issues in the design of next-step tokamak fusion devices. Operating scenarios have been devised to reduce the power incident at the divertor by controlled impurity radiation at the plasma edge and in the scrape-off layer, combined with momentum dissipation in neutral particle interactions. Whether these schemes can be integrated with the stringent core confinement requirements to achieve ignition is yet to be shown, however the more power that the divertor target can safely accept then the less prescriptive are the additional plasma power dissipation requirements. Heat loading at the divertor target is reduced by optimising the divertor geometry, orientating the tiles to spread the power over a larger area. Further reduction in heat loading on the target could be achieved if the scrape-off layer itself could be broadened, ie the radial extent of the power flow channel increased, by increasing the effective radial transport. One approach is by ergodisation of the edge field line structure, in limiter or divertor geometry, but it has yet to be demonstrated whether this is consistent with, for example, high confinement regimes based on an edge transport barrier. Another approach is to stimulate/enhance the production of convective cells in the SOL by the formation of a toroidally-asymmetric potential structure. The resulting ExB convective motions, together with turbulent dissipation, should increase the SOL plasma radial transport and thereby reduce the heat load at the divertor [1]. Several scenarios for achieving the required potential variation have been discussed, one of which is to apply toroidally distributed electrical biasing to the divertor target tiles. In this paper an analysis of this scenario suggests that strong magnetic shear near the X-point will cause significant squeezing of the convection cells in the radial direction and their rapid dissipation, localising the effect to below the X-point. This opens the possibility of reducing the heat load at the divertor target without increasing the radial transport in the portion of the SOL in direct contact with the core plasma, potentially avoiding any confinement degradation. It should be noted that application of electrical biasing is not advocated for power-producer scale devices, because of power consumption 
and other limitations, however it provides a suitable means of testing the common concept of inducing convection by toroidal asymmetry of the boundary.

Electrostatic biasing has been employed in many tokamaks for modifying the edge plasma behaviour, but most of these experiments have concentrated on creating radial and poloidal, rather than toroidal, electric fields ([2] and references therein). JFT-2M have applied nonaxisymmetric divertor biasing in an attempt to drive edge convection, however only 2 out of 14 toroidal segments of the divertor were biased and only slight reductions in heat flux density were observed [3]; no detailed theoretical modelling was reported. On COMPASS-D, a compact tokamak $\left(\mathrm{R}=0.57, \mathrm{a}_{\mathrm{sep}} \sim 0.17 \mathrm{~m}\right)$ with powerful $\mathrm{ECRH}(60 \mathrm{GHz}, \leq 1.5 \mathrm{MW})$, operating in ITER-like single-null divertor configuration, implementation of biasing to the full set of divertor tiles is now programmed for the end of this year (2000). We present below modelling estimates of the bias requirements and predicted effects, together with technical details of the modifications to the divertor.

\section{Theoretical principles}

The fundamental idea is to induce toroidally asymmetric variations of the electrostatic potential in the SOL and set the plasma into convective motion. If the SOL plasma is in good electrical contact with the divertor target then toroidal variations in biasing applied to the tiles can produce the required plasma potential distribution near the target. This toroidal potential distribution is then projected along the field lines into the bulk of the SOL, where it causes ExB drift motion of the ions, which broadens their deposition profile on the divertor target. In a diverted plasma such as that in COMPASS-D, the situation is complicated by the fact that the magnetic field is strongly sheared near the $X$-point. As a result, any magnetic flux tube passing through this region becomes very distorted: its cross-section is squeezed in one direction and elongated in the other direction [4]. This implies that the convection cells created by the biasing cannot penetrate much beyond (above) the $\mathrm{X}$-point since they are squeezed to dimensions smaller than the ion Larmor radius. Induced ExB convection will therefore only take place in the region between the $\mathrm{X}$-point and the deviator, and only this part of the SOL will be broadened. The parallel distance over which broadening is achieved is reduced and becomes of order the shear length, rather than the full connection length.

The magnetic field in the Compass-D divertor is well approximated by the expression for that of a straight field with an X-point in a low-beta plasma,

$$
\mathrm{B}=\hat{\mathrm{z}} \mathrm{B}_{\mathrm{z}}+\hat{\mathrm{z}} \times \nabla \psi=[(\mathrm{x} \hat{\mathrm{x}}-\mathrm{y} \hat{\mathrm{y}}) / \mathrm{L}+\hat{\mathrm{z}}] \mathrm{B}_{\mathrm{z}}
$$

where $\psi=-\mathrm{B}_{\mathrm{z}} \mathrm{xy} / \mathrm{L}$ is the poloidal flux and $\mathrm{L} \sim 1.3 \mathrm{~m}$ is the shear length, (Fig 1). Here (x,y,z) is a Cartesian coordinate system with the origin at the $\mathrm{X}$-point, the $\mathrm{X}$-axis along the divertor leg, and the $\mathrm{z}$-axis in the toroidal direction. The curvature associated with the toroidal field $\mathrm{B}_{\mathrm{z}}$ has been ignored. The field lines are described by $x=x_{0} e^{\left(z-z_{0}\right) / L}, y=y_{0} e^{-\left(z-z_{0}\right) / L}$. Since, on COMPASS-D, the collisional mean-free path exceeds $L$, the field lines are nearly equipotentials and the electrostatic potential is determined by its value at the magnetic footprint,

$$
\phi_{0}(\mathrm{x}, \mathrm{y}, \mathrm{z})=\phi_{0}\left(\mathrm{z}_{0}\right)=\phi_{0}\left(\mathrm{z}-\mathrm{L} \ln \frac{\mathrm{x}}{\mathrm{x}_{0}}\right)
$$


The poloidal projection of the equipotential surfaces, along which ExB motion takes place are shown in Fig 1. Note the strong squeezing of the 'convection cells' approaching the X-point. As already remarked, these cells are wider than the Larmor radius only in the divertor region below the X-point.

To estimate the effect of the induced $\mathrm{ExB}$ drift on ion orbits in the divertor, we note that in the guiding-centre approximation

$$
\frac{\mathrm{d} \psi}{\mathrm{dt}}=\frac{\mathbf{B} \times \nabla \phi_{0}}{\mathrm{~B}^{2}} \cdot \nabla \psi=\frac{\mathrm{d} \phi_{0}}{\mathrm{dz}_{0}}
$$

and since $\mathrm{z}_{0}$ does not change as a result of the drift, d $\psi / \mathrm{dt}$ remains constant along the trajectory. The time an ion spends in the convection region as it travels towards the target is of order $\Delta t=L / v_{T i}$, where $v_{T} \leq\left(T_{i} / m_{i}\right)^{1 / 2}$ the ion thermal speed, and the total deflection of the orbit at the strike point is thus $\Delta \psi \sim \Delta \mathrm{t} \mathrm{d} \psi / \mathrm{dt}=\left(\frac{L}{\mathrm{v}_{\mathrm{Ti}}}\right) \frac{\mathrm{d} \phi_{0}}{\mathrm{dz}_{0}}$ corresponding to

$$
\Delta y=\frac{L \Delta \psi}{B_{z} x_{0}} \sim \frac{L^{2}}{\mathrm{v}_{\mathrm{Ti}} B_{z} x_{0}} \frac{d \phi_{0}}{d z_{0}} \text {. }
$$

Taking for COMPASS-D parameters $\phi_{0}=\Phi_{0}\left(1+\operatorname{coskz} z_{0}\right), \Phi_{0}=40 \mathrm{~V}, \mathrm{k}=\mathrm{N} / 2 \mathrm{R}=16 \mathrm{~m}^{-1}, \mathrm{~N}=16$ $=$ number of tiles, $v_{T i} \sim 5.10^{4} \mathrm{~m} / \mathrm{s}, \mathrm{B}_{\mathrm{z}}=1 \mathrm{~T}$, this gives a mean-square displacement $\left\langle(\Delta \mathrm{y})^{2}\right\rangle \sim$ $7 \mathrm{~cm}$, which significantly exceeds the natural width of about $3 \mathrm{~cm}$ (at the divertor tiles), suggesting that a significant increase could be obtained with modest biasing voltage. (However this also implies that the plasma electrons will perturb the induced electric field, so that strictly speaking a nonlinear calculation is necessary.) Further calculations, which will be published separately, show that significant SOL broadening should occur even if there are turbulent fluctuations in the divertor plasma that are strong enough to destroy guiding-centre orbits.

In order to roughly estimate the power requirements for divertor biasing, suppose that half of the divertor tiles are biased at one voltage, $\phi_{1}$, and the other half at another voltage, $\phi_{2}$, while the plasma at the flux surface under consideration is at the potential $\phi_{0}$. In a typical experiment alternate tiles would be biased, and the other half would be grounded, so that $\phi_{1} \neq 0, \phi_{2}=0$. The current density flowing to the tiles is $j_{k}=n e\left(u-v_{T e} e^{\varphi_{k}-\varphi_{0}}\right)$, where $\varphi_{\mathrm{k}}=\mathrm{e} \phi_{\mathrm{k}} / \mathrm{T}_{\mathrm{e}}$ are normalised potentials, $\mathrm{n}$ is the electron density, $\mathrm{u} \sim \mathrm{v}_{\mathrm{Ti}}$ is the velocity of the ion flow to the target, and $\mathrm{v}_{\mathrm{Te}}=\left(\mathrm{T}_{\mathrm{e}} / 2 \pi \mathrm{m}_{\mathrm{e}}\right)^{1 / 2}$. If the two groups of tiles have the same total area, and if no current flows to other parts of the vessel wall, then $j_{1}+j_{2}=0$ and by eliminating $\varphi_{0}$ from this equation we obtain the current density

and the plasma potential

$$
-j_{1}=j_{2}=e n u \frac{e^{\varphi_{1}}-e^{\varphi_{2}}}{e^{\varphi_{1}}+e^{\varphi_{2}}}
$$

$$
\varphi_{0}=\ln \frac{v_{T e}}{2 u}+\ln \left(e^{\varphi_{1}}+e^{\varphi_{2}}\right)
$$

Thus, positive biasing of one group of divertor tiles can create significant variations of the electrostatic potential inside the plasma, as desired, while negative biasing does not produce a large effect. The current drawn from the tiles is of the order of the ion saturation current j neu. Assuming again alternately biased tiles at one strikepoint, the base-line scenario, the total current driven by the biasing is $\mathrm{I} \sim \pi \mathrm{R} \Delta\left(\mathrm{B}_{\mathrm{P}} / \mathrm{B}_{\mathrm{T}}\right)$ enu . Using representative experimental 
values for COMPASS-D [5]: deuterium, $\mathrm{R} \sim 0.7 \mathrm{~m}$, SOL width $\Delta \sim 3 \times 10^{-2} \mathrm{~m}, \mathrm{~B}_{\mathrm{p}} / \mathrm{B}_{\mathrm{T}} \sim 0.03$, $\mathrm{n} \sim 5 \times 10^{18} \mathrm{~m}^{-3}, \mathrm{~T}_{\mathrm{i}} \sim \mathrm{T}_{\mathrm{e}} \sim 30 \mathrm{eV}$, the total current becomes $\mathrm{I} \sim 100 \mathrm{~A}$. Even a large biasing voltage, $\phi_{1} \sim 5 \mathrm{~T}_{\mathrm{d}} / \mathrm{e} \sim 150 \mathrm{~V}$, gives a relatively low additional power deposited in the SOL, P $\sim 15 \mathrm{~kW}$.

\section{Implementation on COMPASS-D}

The COMPASS-D divertor target consists of inner, middle and outer tile sets (Fig 2) each of 24 (16 broad and 8 narrow) toroidally distributed graphite tiles, and hence is quite suitable in principle for these studies. The two strikepoints and power deposition zones in all configurations are confined to the inner two tile sets, and only these are modified for the biasing. Up to this time, the graphite tiles have been bolted directly to stainless-steel plates mounted on the vacuum vessel, and hence clamped to torus potential. To insulate the tiles for biasing, 125micron thickness PEEK (polyetheretherketone) sheets, moulded to fit the plate contour, are inserted beneath the graphite, and tile fixing bolts are isolated with $\mathrm{Macor}^{\circledR}$ (machinable glass-ceramic) 'top-hats', providing electrical isolation to $1000 \mathrm{~V}$ in air. Kapton ${ }^{\circledR}$ (polyimide) insulated wires are connected to each inner and middle tile and lead outwards through channels machined in the graphite to a circumferential cable duct, and then to multipin vacuum feedthroughs. All insulating surfaces are protected from direct plasma lines of sight by stainless steel or graphite shielding. In nearly all locations the existing tiles are used, machining requirements for modifications being quite limited. All insulated tiles are capable of being individually biased or tied to torus potential, allowing many different configurations to be studied.

Because of machine access limitations, implementation of biasing to all divertor tiles involves removal and subsequent re-installation of the vacuum pumps, auxiliary heating and all major diagnostics, in addition to much demanding in-vessel work. This is resource intensive and estimated to take at least four months. In advance of commencing the full implementation an experimental test of the technical design is being carried out, involving insulating six tiles, localised toroidally, of which four can be electrically biased. A potential problem is posed by halo currents, resulting from vertical displacement events (VDE). Detailed measurements of the intensity and distribution of halo currents in COMPASS-D [6] indicate that peak poloidal currents of up to $0.5 \mathrm{I}_{\mathrm{P}}$, may be expected, suggesting that total currents of up $100 \mathrm{kA}$ could potentially be driven through divertor tiles. Fast acting trips protect the biasing power supply from such halo currents, by switching to an alternative external circuit when excessive currents are detected. However if this alternative is an open circuit then the changes in the poloidal flux at a VDE, together with the lack of a low resistance path could induce excessive voltages on the tiles, leading to insulation breakdown. If the alternative circuit is a low resistance path to the vessel then high currents will flow through the wiring, leading to high $\mathrm{IxB}$ forces. Both of these options are available in the test segment, and measurements will be made of both open circuit induced voltage and short circuit induced currents, to decide on the preferred protection system for the full bias. The effect of $\mathrm{IxB}$ forces is minimised by close coupling the halo return path with the bias wiring, and reacting the forces against each other. Halo current timescales on COMPASS-D are sufficiently short, $\leq 0.5 \mathrm{~ms}$, that resistive heating of the wires should not pose a serious problem.

\section{Summary}

By applying toroidally distributed electrical biasing to divertor target tiles it should be possible to impose a toroidal variation in the near-target SOL plasma potential giving rise to convection cells and broadening of the power deposition zone on the targets. We show that strong magnetic shear near the X-point causes significant squeezing of the convection cells in the radial direction which should lead to their rapid dissipation by resistive and viscous effects. Under realistic conditions therefore, convection essentially does not penetrate above 
Fig 1. Poloidal projection of the divertor region. The $x$-axis is along the divertor leg, from the $X$-point at the origin to the target at $x=x_{0}$, the $y$-axis is along the separatrix between the core plasma and the SOL and the z-axis is in the toroidal direction. The field lines are approximately equipotential in the divertor region. The shaded regions, and the unshaded regions between them. are projections of the divertor tiles along the magnetic field. Thus, if the tiles are biased alternately, these regions will be equipotentials, and $E \times B$ convection will occur, parallel to the $y$ axis, along the lines separating the shaded and unshaded regions. Close to the y-axis these regions become very narrow.

Fig 2. Photograph of the COMPASS-D diventor tiles near the position of the high resolution Langmuir probe array. All inner and middle graphite tiles will be insulated for the full implementation of divertor biasing.
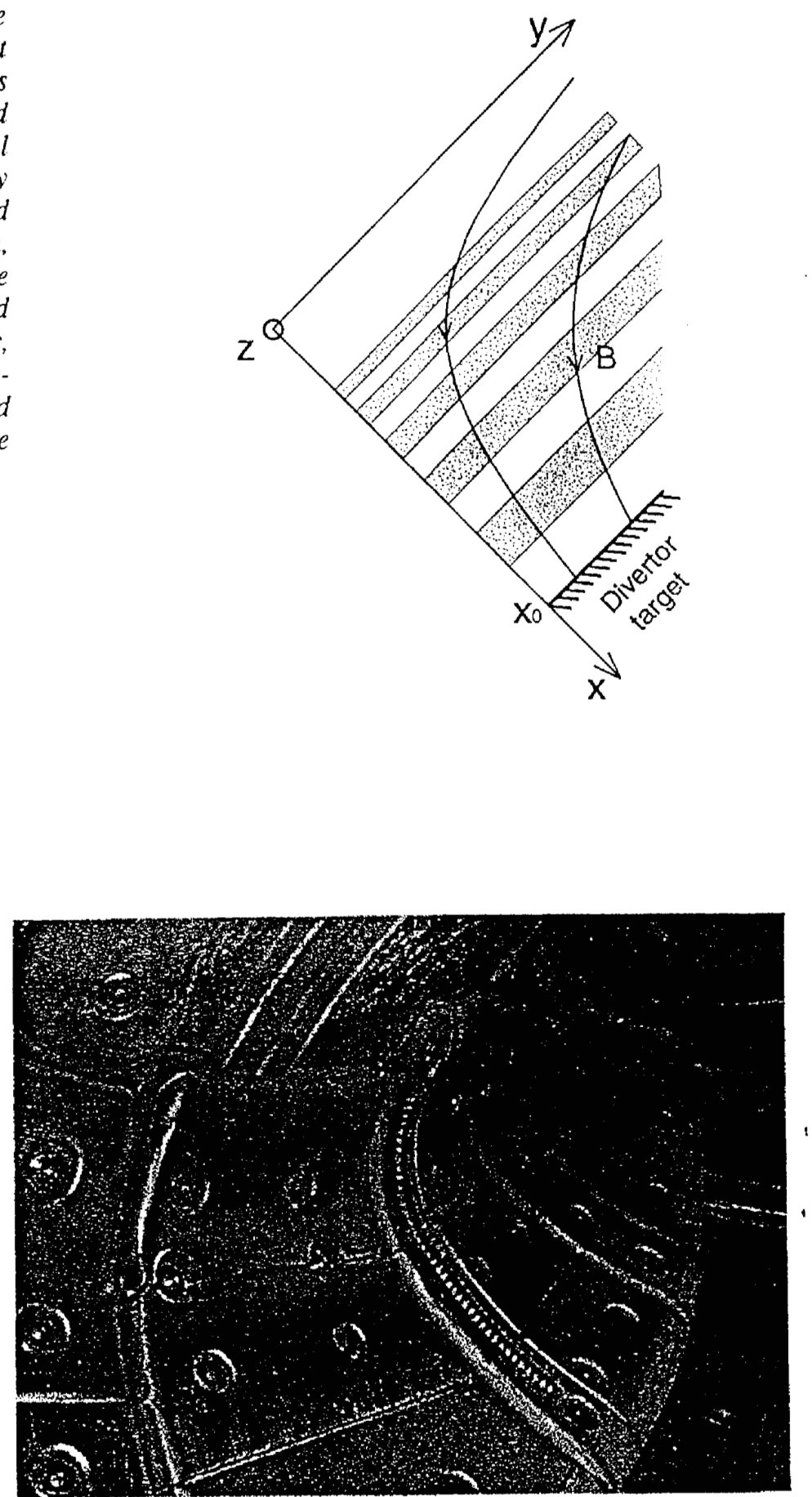
the X-point. Analysis of ion dynamics in a near-collisionless SOL, the case on the COMPASS-D tokamak, shows that significant broadening of the SOL below the X-point should be produced by the planned biasing voltages. This opens the possibility of reducing the heat load at the divertor target without increasing the radial transport in the portion of the SOL in direct contact with the core plasma, potentially avoiding any confinement degradation.

A test of the tile modifications and biasing system is underway on COMPASS-D, which should also determine the best approach to handling halo currents. Implementation of biasing to the full set of divertor tiles, with the flexibility to explore many tile configurations, is now programmed for the end of this year. COMPASS-D has a comprehensive set of edge diagnostics including a high resolution divertor Langmuir probe array, a reciprocating Langmuir probe and helium jet spectroscopy and in particular should be able to resolve different changes to the target and SOL plasmas.

This work was performed under the auspices of the U.S. Department of Energy by the University of California, Lawrence Livermore National Laboratory under contract No. W-7405-Eng-48.

\section{References}

[1] R.H. Cohen and D.D. Ryutov, Nucl. Fusion 37, 621 (1997)

[2] R.R.Weynants and G.van Oost, Plasma Phys. Control. Fusion 35 (1993) B 177

[3] J. Hara, Y. Uesugi, Y. Miura, H. Kawashima, J Nucl Mats 241-243, 338 (1997)

[4] D. Farina, R. Pozzoli, D. Ryutov, Nucl. Fusion 33, 1351 (1993).

[5] C.G. Silva, S.J. Fielding, K.B. Axon, M.G. Booth, Plasma Phys. Control. Fusion 40 (1998) 1159

[6] G.G. Castle, A.W. Morris, D. Gates, M. Valovic, Proc $23^{\text {rd }}$ EPS Conference on Controlled Fusion and Plasma Physics, Kiev, 1996, Vol I, 139 